\title{
Identifying, Analyzing, and Visualizing Diagnostic Paths for Patients with Nonspecific Abdominal Pain
}

\author{
Goutham Rao ${ }^{1}$ Katherine Kirley ${ }^{2}$ Paul Epner ${ }^{3}$ Yiye Zhang ${ }^{4}$ Victoria Bauer ${ }^{5}$ Rema Padman ${ }^{6}$ \\ Ying Zhou ${ }^{5}$ Anthony Solomonides ${ }^{5}$
}

\footnotetext{
${ }^{1}$ Department of Family Medicine and Community Health, University Hospitals of Cleveland, Case Western Reserve University, Cleveland, Ohio, United States

2 American Medical Association, Chicago, Illinois, United States

${ }^{3}$ Society to Improve Diagnosis in Medicine, Evanston, Illinois, United States

${ }^{4}$ Department of Healthcare Policy and Research, Weill Cornell Medicine, New York, New York, United States

${ }^{5}$ Ambulatory Primary Care Innovations Group, NorthShore University HealthSystem, Evanston, Illinois, United States

${ }^{6}$ Heinz College, Carnegie Mellon University, Pittsburgh, Pennsylvania, United States
}

Appl Clin Inform 2018;9:905-913.

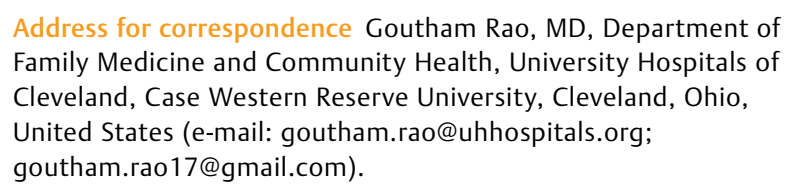

Address for correspondence Goutham Rao, MD, Department of Family Medicine and Community Health, University Hospitals of Cleveland, Case Western Reserve University, Cleveland, Ohio, United States (e-mail: goutham.rao@uhhospitals.org; goutham.rao17@gmail.com).

\section{Abstract}

Keywords

- diagnosis

- primary care

- electronic health records

- abdominal pain
Background Diagnosis is complex, uncertain, and error-prone. Symptoms such as nonspecific abdominal pain are especially challenging. A diagnostic path consists of diagnostic steps taken from initial presentation until a diagnosis is obtained or the evaluation ends for other reasons. Analysis of diagnostic paths can reveal patterns associated with more timely and accurate diagnosis. Visual analytics can be used to enhance both analysis and comprehension of diagnostic paths.

Objective This article applies process-mining methods to extract and visualize diagnostic paths from electronic health records (EHRs).

Methods Patient features, actions taken (i.e., tests, referrals, etc.), and diagnoses obtained for 501 adult patients (half female, half $\geq 50$ years of age) presenting with abdominal pain were extracted from an EHR database to construct diagnostic paths from a hospital system in suburban Chicago, Illinois, United States. A stable diagnosis was defined as the same diagnosis recorded twice in a 12-month period; a working diagnosis was recorded only once. Three different types of path visualizations were obtained.

Results A stable diagnosis was obtained in $63(13 \%)$ patients after 12 months. In 271 (54\%) patients, a working diagnosis was obtained. Mean path duration was 145.3 days (standard deviation, 195.1 days). These 63 patients received 75 stable diagnoses. Conclusion Structured EHR data can be used to construct diagnostic paths to gain insight into diagnostic practices for complaints such as abdominal pain.

\section{Background and Significance}

The National Academy of Medicine describes diagnostic errors as a blind spot in the delivery of quality of health care. ${ }^{1}$ Diagnosis is often characterized by complexity, uncertainty, and error. ${ }^{2}$ Not surprisingly, diagnostic practices are highly variable. ${ }^{3,4}$ Such variation may only be partially explained by different patient characteristics and presentations. The residual variation may be the result of differing individual physician heuristics and experiences. ${ }^{5,6}$ Some diagnostic practices are received

July 25,2018

accepted after revision

October 25, 2018 (c) 2018 Georg Thieme Verlag KG Stuttgart . New York
DOI https://doi.org/

10.1055/s-0038-1676338. ISSN 1869-0327. 
associated with delayed or incorrect diagnosis, unnecessary expense, overtreatment, and substantial harm. ${ }^{7}$

Guidance about best diagnostic practices is scarce. Few guidelines, for example, provide recommendations on how best to carry out a diagnostic evaluation for common specific patient complaints. Nonspecific complaints such as nonspecific abdominal pain and dizziness are especially challenging for primary care. ${ }^{8}$ Nonspecific complaints are associated with significant diagnostic variation, high rates of diagnostic error, and frequent nondiagnosis. ${ }^{2,9-13}$ An important step in improving diagnosis for common complaints is to gain a better understanding of current diagnostic practices.

We have previously defined the term diagnostic path as a systematic approach to describing the steps taken for diagnostic evaluation of a complaint after initial presentation of a patient until either a stable diagnosis is achieved (i.e., a diagnosis which becomes the basis for treatment) or the patient and physician choose to end the diagnostic evaluation without obtaining a diagnosis (e.g., pursuing symptom management without further diagnostic evaluation). ${ }^{14}$ Although it is clear that evidence of diagnostic reasoning will be largely in the textual note, the problem we have addressed is to determine how much may be discerned from the sequence of discrete events in the record through methods borrowed from the process mining and phenotyping paradigms. We have developed a methodology for extracting the data necessary to construct diagnostic paths for a sample of patients from a repository of electronic health records (EHRs) data and applied it to the problem of nonspecific abdominal pain. Furthermore, we have developed a novel method for visualizing diagnostic paths which we believe, when applied to diagnostic paths of a large number of patients, is useful to facilitate understanding by clinicians and patients, and also for analysis of patterns of diagnostic practices. We have previously described the technical aspects of our work in a detailed paper. ${ }^{15}$ For this article, we developed two visualizations from a small sample to provide a proof of principle for the methodology. We emphasize the clinical aspects and clinical significance of the application of diagnostic paths to abdominal pain. We believe diagnostic paths are useful both for understanding diagnostic practice variation and to identify best diagnostic practices associated with more timely and accurate diagnosis, and less resource-intensive diagnostic evaluation.

Abdominal pain in primary care is an ideal test case to apply the concept of diagnostic paths, as it is very common, comprising the reason for visit among $1.5 \%$ of all patients presenting in primary care. ${ }^{16}$ It is associated with both relatively benign (e.g., gastroesophageal reflux disease [GERD]) and serious conditions (e.g., cancer), making it important to weigh the risks of missing a serious diagnosis against unnecessary testing. Diagnostic approaches to abdominal pain are highly variable. ${ }^{15,17-20}$ In over half of patients a diagnosis is not reached. ${ }^{21}$ Moreover, evidencebased guidance on best approaches to abdominal pain in primary care is unavailable. The few published guidelines that do exist are focused upon the emergency room setting. $^{22}$

\section{Objective}

Our primary objective was to apply the concept of diagnostic paths to structured EHR data, including both quantitative analysis and visualization. We chose abdominal pain as a test case for the reasons cited above. In a previous paper, we emphasized the technical aspects of visualization. ${ }^{15}$ In this article, we focus upon how analysis of diagnostic paths can be used to better understand diagnosis in patients with abdominal pain, and can eventually serve as a useful source of diagnostic guidance.

\section{Methods}

\section{Data Sources/Extraction and Key Definitions}

Two authors first independently identified structured data fields from EHR records relevant to abdominal pain through manual chart review of 25 patients. We also completed a quantitative analysis of the most common tests, prescriptions, procedures, and referrals provided or performed in patients with abdominal pain in primary care settings in our health system. Based on the independent chart review assessment which was observed by a third member of the team and quantitative analysis and based on counts of completeness, the research team iterated the process to construct a consensus data extraction template relevant to abdominal pain (see - Supplementary Material, available in the online version).

The relevant EHR data were extracted from the Enterprise Data Warehouse (EDW), a comprehensive data repository of over 1 million patients extracted primarily from the Epic Systems EHR (Epic Systems, Verona, Wisconsin, United States). From among approximately 12,000 patients who presented for the first time in 2011 or 2012 in primary care (family medicine or internal medicine) with a chief complaint/reason for visit of abdominal pain (as well as two related structured terms, "epigastric pain" and "stomach pain") with no encounters or referrals for abdominal pain in the prior 12 months, we randomly selected 501 patients based on these criteria: (1) 50\% female and (2) 50\% with age $\geq 50$ years. Our purposive sampling was chosen to reflect the very different etiologies of abdominal pain among men and women, and older and younger patients. All encounters and activity for each patient for a period of up to 12 months after the index encounter were analyzed.

We defined a "stable diagnosis" as a specific problemrelated diagnosis which is recorded separately (i.e., not just carried over as evidenced by the relevant timestamp) on at least two separate encounters during the 12-month study period. For example, consider a patient with abdominal pain who is not given a specific abdominal pain-related diagnosis after two encounters, but a diagnosis of GERD is recorded at the third encounter, peptic ulcer disease at the fourth encounter, and GERD is recorded at the fifth encounter, the patient's "stable diagnosis" is considered as GERD. The assumption is that because the physician has recorded the same specific diagnosis twice, it has become the basis for treatment. We defined a "working" diagnosis as a specific 
diagnosis which is recorded only once during the study period. A patient can have more than one working or stable diagnosis during the study period. For patients with stable diagnoses, the duration of a diagnostic path is defined as the length of time from the first presenting encounter to the point at which the first of two identical problem-specific diagnoses are recorded. These definitions have been conceived only to operationalize the application of diagnostic paths to EHRs, and may not accurately reflect physicians' thinking. The definitions are not rigid and the endpoint and duration of a diagnostic path can be defined in other ways. A "working" or "stable" diagnosis may be made in any clinical (rather than just primary care) setting. It should also be noted that there is no distinction between chronic and acute diagnoses, although that may be identifiable from the record through temporal analysis: for an acute condition, the two independent diagnoses may be expected to have occurred closer together in time.

\section{Statistical Analysis}

Descriptive statistics were summarized as mean \pm standard deviation (SD) for continuous variables and $N(\%)$ for categorical variables. Normality was evaluated using graphical methods and the Shapiro-Wilk test. Continuous variables were compared using the two-sample t-test or analysis of variance for normally distributed data, or Wilcoxon ranksum test and Kruskal-Wallis test for skewed data. Categorical variables were compared using the chi-squared test or Fisher's exact test. All data were analyzed using SAS v9.3 (SAS Institute, Cary, North Carolina, United States). $p$-Values of $<0.05$ were considered significant.

\section{Visualization Methods}

Understanding diagnostic paths for individual or a large number of patients in a way that is meaningful to clinicians is challenging. Visual analytics addresses this challenge. ${ }^{23}$ When applied to individual diagnostic paths or paths from thousands of patients, visualization promotes easier data consumption. Analysis based on visualization of thousands of paths can also provide insights that are complementary to a purely quantitative statistical approach. We applied a visual analytics methodology to the construction of diagnostic paths. ${ }^{24}$ There are two essential steps: transformation of multifactorial sources of clinical information into a pathform data structure, and identification of common path patterns among clusters of similar paths. The first step is critical in summarizing the steps taken to diagnose patients such that patients who received similar approaches can be identified as clusters. We do so by constructing encounter identifiers for each encounter, where each identifier encompasses multifactorial information. The resulting path-form data allow us to identify common data patterns in all, or in a subset, of the data by examining transition frequencies. We refer to an encounter taking place first in the path as source and the successor as target. For example, given a path with encounter identifiers V3-V2-V2-V5, there are three transitions: (V3, V2), (V2, V2), and (V2, V5), where the source is V3 and target is $\mathrm{V} 2$ in the first transition. To ensure that common patterns occur in the data beyond a certain probability, we define a measure:

$$
\text { weight } t_{i j}=\frac{\#\left(N_{j}=\text { target } \mid N_{i}=\text { source }\right)}{\#\left(N_{i}=\text { source }\right)},
$$

where

$$
\#\left(N_{i}=\text { source }\right)
$$

is the number of times $N_{i}$ appears as a source, and

$$
\#\left(N_{j}=\text { target } \mid N_{i}=\text { source }\right)
$$

is the number of times $N_{j}$ appears as a target given $N_{i}$ is the source. Weight is therefore the conditional probability that $N_{i}$ will transition to $N_{j}$. We also capture the difference in days between source and target as time gap. If the same transition of visits has more than one time gap observed in multiple paths, we take the average of all time gaps for a transition. Our visualization methods are described in additional detail in a paper by Zhang et al. ${ }^{24}$

\section{Results}

\section{Characteristics of Patients}

We divided our set of 501 patients into those with "stable," "working," and "no diagnosis" after 12 months. Patient characteristics are summarized in - Table 1. Sixty-three (13\%) patients had a stable diagnosis, 271 (54\%) had a working diagnosis, and 167 (33\%) had no specific diagnosis. Patients without a diagnosis were younger, less likely to report allergy, and less likely to have recorded past medical problems.

\section{Diagnostic Actions}

We divided actions taken into broad categories which included laboratory testing, imaging, prescriptions, and referral. We were especially interested in actions taken during the first encounter for abdominal pain, as we found (to be discussed later) that among those with stable diagnoses, the diagnosis was often made at that encounter. We compared patients with a stable diagnosis to those with working and no diagnoses. There were no significant differences among patients with stable, working, and no diagnosis in terms of the rate of prescription of any medication at the first encounter $(68.25,75.28$, and $67.66 \%$, respectively, $p=0.18$ ). Patients with a stable diagnosis or working diagnosis were more likely to be prescribed any medication during the 12-month follow-up period (98.41 and $97.42 \%$, respectively) versus just $80.84 \%$ of patients with no diagnosis $(p<0.01)$. A total of $77.78 \%$ of patients with a stable diagnosis were prescribed a gastrointestinal drug in the 12month follow-up period compared with 57.93 and 29.94\% of patients with a working and no diagnosis, respectively $(p<0.01)$.

Note that $20.63 \%$ of patients with a stable diagnosis were referred to a gastroenterologist compared with $22.14 \%$ of patients with a working diagnosis and $13.17 \%$ of patients with no diagnosis (nonsignificant differences). A total of 
Table 1 Characteristics of patients with stable, working, and no diagnosis

\begin{tabular}{|c|c|c|c|c|}
\hline Characteristic & $\begin{array}{l}\text { Patients with } \\
\text { stable diagnosis } \\
(N=63)\end{array}$ & $\begin{array}{l}\text { Patients with } \\
\text { working diagnosis } \\
(N=271)\end{array}$ & $\begin{array}{l}\text { Patients with } \\
\text { no diagnosis } \\
(N=167)\end{array}$ & $\begin{array}{l}p \text {-Value for } \\
\text { differences }\end{array}$ \\
\hline \multicolumn{5}{|l|}{ Demographics } \\
\hline Age (mean, SD) & $53.5 \pm 16.95$ & $55.49 \pm 17.28$ & $47.21 \pm 14.92$ & $<0.01$ \\
\hline BMI (mean, SD) & $27.7 \pm 5.51$ & $27.76 \pm 5.39$ & $27.22 \pm 5.87$ & 0.61 \\
\hline Sex - Female (\%) & $31(49.21)$ & $143(52.77)$ & $76(45.51)$ & \multirow[t]{2}{*}{0.33} \\
\hline Sex - Male (\%) & $32(50.79)$ & $128(47.23)$ & $91(54.49)$ & \\
\hline \multicolumn{5}{|l|}{ Race } \\
\hline African American (\%) & $1(1.59)$ & $9(3.32)$ & $5(2.99)$ & \multirow[t]{4}{*}{0.60} \\
\hline Asian (\%) & $2(3.17)$ & $11(4.06)$ & $13(7.78)$ & \\
\hline White Caucasian (\%) & $42(66.67)$ & $172(63.47)$ & $107(64.07)$ & \\
\hline Other (\%) & $18(28.57)$ & $79(29.15)$ & $42(25.15)$ & \\
\hline \multicolumn{5}{|l|}{ Ethnicity } \\
\hline Hispanic (\%) & $6(9.52)$ & $21(7.75)$ & $14(8.38)$ & \multirow[t]{2}{*}{0.89} \\
\hline Non-Hispanic (\%) & $57(90.48)$ & $250(92.25)$ & $153(91.62)$ & \\
\hline \multicolumn{5}{|l|}{ Patient's preferred language } \\
\hline English (\%) & $61(96.8)$ & $260(95.94)$ & $160(96.97)$ & \multirow[t]{2}{*}{0.60} \\
\hline Not English (\%) & $2(3.2)$ & $11(4.06)$ & $5(3.03)$ & \\
\hline \multicolumn{5}{|l|}{ Medical history } \\
\hline Any reported allergy (\%) & $30(47.62)$ & $133(49.08)$ & $59(35.33)$ & 0.02 \\
\hline $\begin{array}{l}\text { Any reported past medical } \\
\text { history on problem list (\%) }\end{array}$ & $44(69.8)$ & $190(70.11)$ & $95(56.89)$ & 0.02 \\
\hline $\begin{array}{l}\text { Any reported past surgical } \\
\text { history on problem list (\%) }\end{array}$ & $38(60.32)$ & $160(59.04)$ & $94(56.29)$ & 0.80 \\
\hline Any reported family medical history (\%) & $44(69.84)$ & $179(66.05)$ & $111(66.47)$ & 0.85 \\
\hline
\end{tabular}

Abbreviations: BMI, body mass index; SD, standard deviation.

$9.52 \%$ of patients with a stable diagnosis were referred to an obstetrician/gynecologist compared with $2.95 \%$ of those with a working diagnosis and $1.20 \%$ of patients with no diagnosis $(p<0.01)$.

There were no significant differences among patients with a stable, working, or no diagnosis in the percentage who had a laboratory or imaging test ordered or procedure at the first encounter (53.97, 52.4, and 49.1\%, respectively, $p=0.731$ ). However, over 12 months, $98.41 \%$ of patients with a stable diagnosis, $94.83 \%$ of patients with a working diagnosis, and $80.84 \%$ of patients with no diagnosis had some type of laboratory or imaging test or procedure ordered $(p<0.01)$.

A complete blood count $(\mathrm{CBC})$ was the most commonly ordered laboratory test at the first encounter among all three groups of patients $(47.62,36.9$, and $40.72 \%$ among those with stable, working, and no diagnosis, respectively, nonsignificant differences). In general, there were few differences among the three groups of patients in terms of rates of ordering specific tests and imaging studies at the first encounter. There were, however, significant differences when all encounters in the 12-month study period were considered. Almost all specific tests were more likely to be ordered among those with a stable diagnosis (-Table 2).

\section{Stable Diagnoses}

The 63 patients with stable diagnoses in our data set had a total of 75 different diagnoses ( - Table 3 ). A single patient can have $\geq 1$ stable diagnoses recorded in the study period. Among 75 diagnoses, the most common diagnoses were gastrointestinal $(n=43,57 \%)$, followed by urological ( $n=25,33 \%)$ and gynecological $(n=7,9 \%)$.

\section{Duration and Frequency of Visits}

The mean duration to a stable diagnosis in our sample was 145.3 days (SD, 195.1 days), with a median duration of 56 days. Gastrointestinal diagnoses were made in the shortest time (125.35 days, SD, 209.94 days, median 14 days). Mean times for gynecological and urological diagnoses were 143.29 (SD, 223.27, median 29) and 180.16 (SD, 160.46, median 154) days, respectively.

Path duration was longer for women than men (159.25 vs. 129.34 days, $p=0.046$ ). Patient age and path duration were significantly correlated (Pearson's correlation coefficient 0.340, $p<0.01$ ).

Path duration may not reflect the effort required to obtain a "stable" diagnosis. We therefore also calculated the frequency of outpatient office visits among those with stable 
Table 2 Laboratory tests and imaging over 12-month follow-up period

\begin{tabular}{|c|c|c|c|c|}
\hline Test/Imaging study & $\begin{array}{l}\text { Patients with stable } \\
\text { diagnosis } \\
(N=63)\end{array}$ & $\begin{array}{l}\text { Patients with } \\
\text { working diagnoses } \\
(N=271)\end{array}$ & $\begin{array}{l}\text { Patients with } \\
\text { no diagnosis } \\
(N=167)\end{array}$ & $\begin{array}{l}p \text {-Value for } \\
\text { differences }\end{array}$ \\
\hline \multicolumn{5}{|l|}{ Tests } \\
\hline Serum amylase & $15(23.81 \%)$ & $34(12.55 \%)$ & $18(10.78 \%)$ & 0.029 \\
\hline Basic metabolic panel & $25(39.68 \%)$ & $88(32.47 \%)$ & $28(16.77 \%)$ & 0.029 \\
\hline Clostridium difficile colitis & $8(12.70 \%)$ & $15(5.54 \%)$ & $7(4.19 \%)$ & 0.048 \\
\hline Complete blood count & $59(93.65 \%)$ & $219(80.81 \%)$ & $118(70.66 \%)$ & $<0.01$ \\
\hline Urinalysis & 40 (63.49\%) & $152(56.09 \%)$ & $64(38.32 \%)$ & $<0.01$ \\
\hline Urine culture & $25(39.68 \%)$ & $74(27.31 \%)$ & $12(7.19 \%)$ & $<0.01$ \\
\hline Complete metabolic panel & $54(85.71 \%)$ & $217(80.07 \%)$ & $109(65.27 \%)$ & $<0.01$ \\
\hline Helicobacter pylori testing & $15(23.81 \%)$ & $34(12.55 \%)$ & $11(6.59 \%)$ & $<0.01$ \\
\hline Liver enzymes & $22(34.92 \%)$ & $52(19.19 \%)$ & $16(9.58 \%)$ & $<0.01$ \\
\hline \multicolumn{5}{|l|}{ Imaging } \\
\hline $\begin{array}{l}\text { Computed tomography (CT) scan } \\
\text { of abdomen and/or pelvis }\end{array}$ & $28(44.44 \%)$ & $76(28.04 \%)$ & $24(14.37 \%)$ & $<0.01$ \\
\hline X-ray abdomen & $10(15.87 \%)$ & $9(3.32 \%)$ & $1(0.6 \%)$ & $<0.01$ \\
\hline Ultrasound of abdomen or pelvis & $24(38.10 \%)$ & $53(19.56 \%)$ & $31(18.56 \%)$ & $<0.01$ \\
\hline
\end{tabular}

diagnoses. The mean number of office visits in this group was 2.23 , with a SD of 4.08 .

\section{Visualization Results}

Among the 63 patients with stable diagnoses, we visualized 51 distinct paths. - Fig. 1 is a visualization of diagnostic paths for the 63 patients with stable diagnoses. The size of each node and the thickness of each arrow are proportional to the number of patients who follow a specific path segment. The upper graphic of the figure is very complex. When the figure is limited to nodes which include a minimum of two patients, and actions are grouped into larger categories, the lower graphic of the figure is the result, and is easier to digest. The figure includes classification of specific actions into categories including prescription of central nervous system-acting drugs, prescription of other classes of drugs, gynecological or urological referrals, and imaging tests of any kind. Note that in a significant proportion of patients (10/63,16\%), a diagnosis was made immediately after the index encounter with no further evaluation.

-Fig. 2 is a different type of visualization, which shows three very different diagnostic paths for three patients eventually diagnosed with GERD.

\section{Discussion}

Abdominal pain is a common and challenging problem in primary care and is associated with significant diagnostic variation. We successfully applied the concept of diagnostic paths to an EHR-derived data repository by extracting, analyzing, and visualizing the necessary data for 501 patients with nonspecific abdominal pain. Ours is not the first effort to visualize care patterns from EHR data, ${ }^{25,26}$ but is the first to apply visual analytics to a common primary care problem. We were able to successfully create visualizations of paths of all patients in whom a stable diagnosis was obtained, visualize the paths of individual patients, and provide a summary visualization of common paths which includes a time scale. Through visualization, a clinician can see the diagnostic path of an individual patient to learn how a diagnosis was reached, or can see patterns of diagnostic paths which may reveal how his or her colleagues are approaching a common problem.

One of our most important findings is that our definition of "stable diagnosis" leaves $87 \%$ of patients undiagnosed after a year. Even the less restrictive "working diagnosis" endpoint leaves $33 \%$ undiagnosed. This finding is consistent with past studies. van der Weijde et al report that in more than half of all cases of abdominal pain in primary care a specific diagnosis is not reached. ${ }^{21}$ A 2005 study reported that even among elderly patients presenting with abdominal pain to emergency rooms and followed-up 2 weeks later, a specific diagnosis had not been established in $14.8 \%$ of cases. ${ }^{20}$ In our study, the low rates of both stable and working diagnoses may not only reflect a failure to reach a diagnosis, but also cases in which patients' symptoms resolved either spontaneously or through empiric treatment, or patients who were lost to follow-up, or seen elsewhere. Our structured quantitative data are not able to distinguish among these possibilities.

It takes an average of nearly 5 months to make a specific diagnosis based on our definition of "stable" diagnosis. This duration is somewhat misleading, as in a significant proportion of patients (16\%) a stable diagnosis is made based upon a single office visit with no further evaluation.

Although a wide variety of laboratory tests, imaging studies, and referrals are made among the patients studied, 
Table 3 Stable diagnoses $(n=63)$

\begin{tabular}{|c|c|}
\hline Diagnosis & Frequency (\%) \\
\hline \multicolumn{2}{|l|}{ Gastrointestinal problems } \\
\hline Esophageal reflux/heartburn (GERD) & $10(15.9)$ \\
\hline Diverticulitis & $4(6.3)$ \\
\hline Cholelithiasis & $4(6.3)$ \\
\hline Dyspepsia & $3(4.8)$ \\
\hline Gastritis/gastroduodenitis & $2(3.2)$ \\
\hline Clostridium difficile colitis & $2(3.2)$ \\
\hline Cholecystitis & $2(3.2)$ \\
\hline Anal fissure & $2(3.2)$ \\
\hline Inguinal hernia & $2(3.2)$ \\
\hline Noninfectious gastroenteritis & $1(1.6)$ \\
\hline Cholangitis & $1(1.6)$ \\
\hline Ventral hernia & $1(1.6)$ \\
\hline Irritable colon & $1(1.6)$ \\
\hline Hepatitis & $1(1.6)$ \\
\hline Intestinal abscess & $1(1.6)$ \\
\hline Nonspecific intestinal obstruction & $1(1.6)$ \\
\hline Enterostomy complication & $1(1.6)$ \\
\hline Infectious diarrhea & $1(1.6)$ \\
\hline Intussusception & $1(1.6)$ \\
\hline Regional enteritis & $1(1.6)$ \\
\hline Appendicitis & $1(1.6)$ \\
\hline \multicolumn{2}{|l|}{ Gynecological problems } \\
\hline Menorrhagia & $2(3.2)$ \\
\hline Ovarian cyst & $1(1.6)$ \\
\hline Noninflammatory disease of ovary & $1(1.6)$ \\
\hline Noninflammatory disease of vagina & $1(1.6)$ \\
\hline Nonspecific menstrual disorder & $1(1.6)$ \\
\hline Postmenopausal bleeding & $1(1.6)$ \\
\hline \multicolumn{2}{|l|}{ Urinary tract problems } \\
\hline Urinary tract infection & $15(23.8)$ \\
\hline Nephrolithiasis & $8(12.7)$ \\
\hline Bladder stone & $2(3.2)$ \\
\hline
\end{tabular}

Abbreviation: GERD, gastroesophageal reflux disease.

a consistent finding is that more diagnostic services are provided to those in whom a stable diagnosis is achieved. A simple, but potentially misleading conclusion may be that more testing is necessary to obtain a diagnosis. Equally, patients who are better engaged in the health system may be more likely receive and/or to follow-up with recommended testing. Or clinicians who are aware that a diagnosis may not be reached in a large proportion of patients may actually order diagnostic tests only in patients for whom a specific diagnosis is more likely.

Our results are subject to several limitations. First, our definitions of "stable diagnosis" and path duration were based only on consensus among the research team. Nevertheless, our findings, and even more so, our methods have the potential to inform a review of these definitions. Second, there is little doubt that patient characteristics and details of presentation are important drivers of diagnostic paths. Some of these data are included in free-text notes in the EHR. We did carry out manual chart reviews on a small scale to validate structured field data extracted from the EDW. Supplementing structured field data with free-text notes, however, proved both impractical and not valuable because physicians' notes were frequently missing key elements of the clinical evaluation. It is also unclear to what extent physicians' notes accurately represent diagnostic thinking. For these reasons, we limited our analysis to data from structured fields. While the diagnostic process is guided by the evolving individual clinical features with which individual patients present, we believe analysis on a large scale will minimize problems such as attribution or the absence of free-text notes, since large numbers of patients with broadly similar characteristics will be analyzed.

We are also uncertain to what extent the large proportion of patients in whom no diagnosis is obtained represents a diagnostic failure, or simply patients whose symptoms have resolved, or through frustration or for other reasons sought diagnostic help outside our health system.

Finally, as noted above, diagnostic paths as extracted from the EHRs may not accurately reflect physicians' thinking. This is closely related to the problem of attribution which we encountered during our manual chart reviews. For example, if a patient with a primary complaint of abdominal pain also suffers from anemia due to menorrhagia, and a $C B C$ is ordered, to which problem is the test attributable? In some cases, it is not possible to know.

Our next goal is to apply our methodology to thousands of patients, in which more robust patterns of diagnostic practices will be identifiable for abdominal pain and other challenging problems. Visualization will also be useful in easily identifying patterns among diagnostic paths when a large number of patients is analyzed. Our visualizations were based on a relatively small number of patients and performed primarily to apply our visualization methodology. We acknowledge that, even on a very large scale, identifying patterns of diagnostic paths can only tell us which patterns are best among those being practiced, rather than what an optimal path, or candidate pathway, may be, although it should serve to eliminate the weakest paths from consideration.

\section{Conclusion}

Diagnostic paths can be successfully identified and visualized from structured EHR data. In a sample of patients with the challenging primary care problem of abdominal pain, only a small proportion received a stable diagnosis after 12 months. Patients who receive more diagnostic services are more likely to also receive a diagnosis. In the majority of cases, a diagnosis is obtained quickly after one visit, or after several months. Our study represents an important beginning in the application of the concept of diagnostic paths to EHR data. We believe that we will be able to identify patterns 

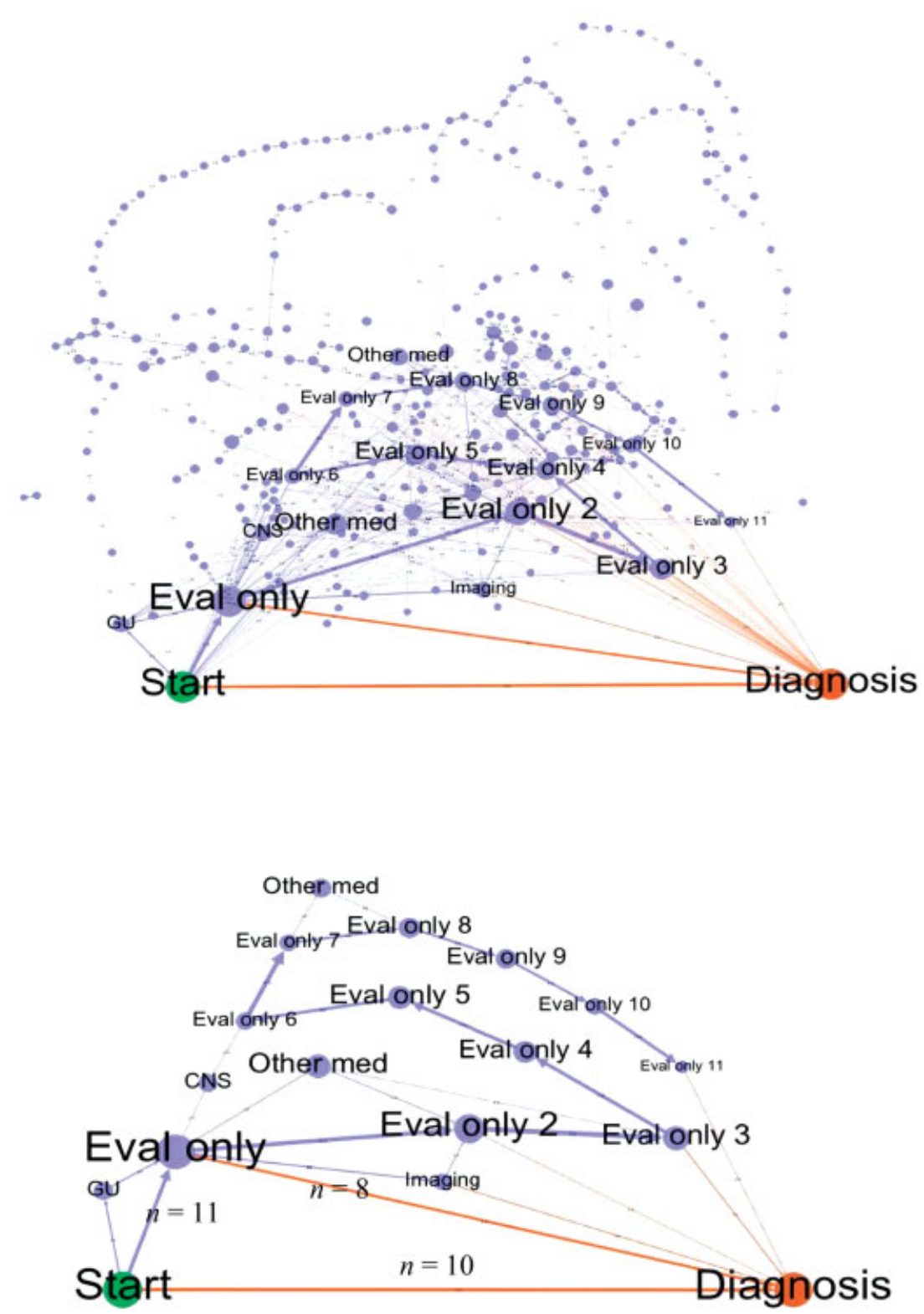

Fig. 1 Diagnostic paths of patients in whom a stable diagnosis was obtained. Top: Complete diagnostic paths for 63 patients. The size of nodes and thickness of arrows is proportion to the number of patients who follow each path segment. Bottom: Diagnostic paths of patients with stable diagnosis including nodes with $\geq 2$ patients. Eval only: physician completed clinical evaluation only with no other actions. GU: gynecologic urology referral. Imaging: imaging order. CNS: prescription of central nervous system drug. Prescription, prescriptions of medications from other classes. Laboratory tests were analyzed individually and not grouped into categories (e.g., hematology, chemistry). A large variety of different laboratory tests were ordered, with only a limited number of different tests in each category. Only actions which occurred among $\geq 2$ patients are included, which is why individual laboratory tests or test categories do not appear.

more likely to be associated with timely, accurate, and resource-efficient diagnosis. These preferred patterns could form the basis of diagnostic guidance, which today, is largely based only on consensus recommendations.

\section{Clinical Relevance Statement}

Diagnosis, especially among patients with common nonspecific complaints such as abdominal pain, is extremely challenging for physicians who also lack guidance on best diagnostic practices. Analyzing and visualizing diagnostic paths on a large scale, for which our study lays the foundation, has the potential to reveal more timely, accurate, and resource-efficient diagnostic practices.

\section{Multiple Choice Questions}

1. Which statement best describes a "diagnostic path"?

a. A guideline on best diagnostic practices designed for practicing physicians.

b. A description of the steps taken from first presentation to diagnosis. 


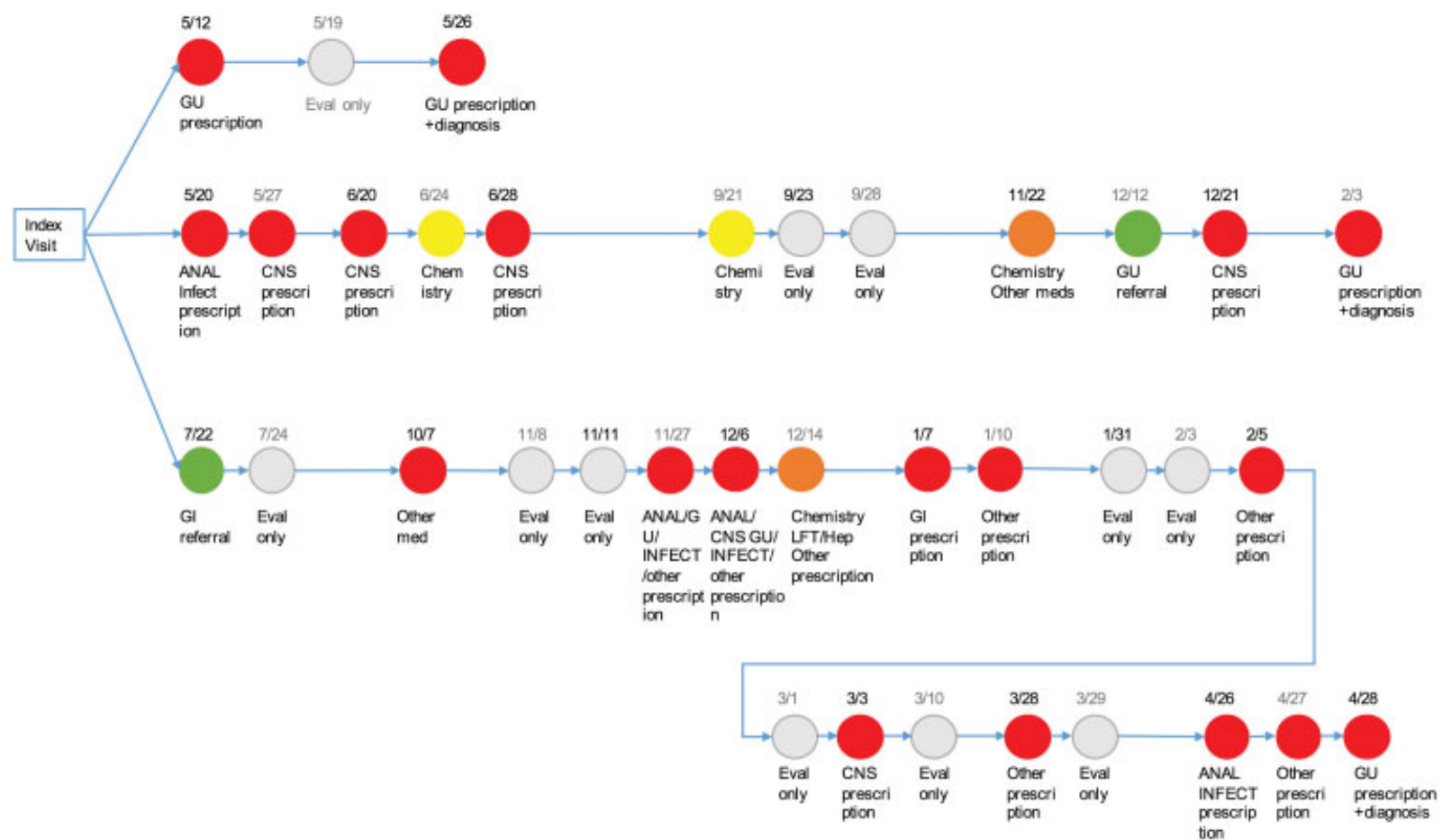

Fig. 2 Paths to gastroesophageal reflux disease (GERD). Three interesting diagnostic paths to a diagnosis of GERD with a timeline. Arrows emanating from index visit indicate everything that took place at that visit (first circle after index visit) and thereafter according to the timeline. Gray circle, encounter with clinical evaluation only; green circle, encounter with clinical evaluation plus referral; red circle, encounter with prescription; yellow circle, encounter with laboratory testing; orange circle, encounter with laboratory testing plus prescription. Medications are classified by therapeutic category: GI, gastrointestinal; CNS, central nervous system; Infect, anti-infective; GU, genitourinary; analgesic, analgesic (e.g., oxycodone); other, other medication category.

c. A description of a patient's preferences for diagnosis throughout their interaction with the health care system.

d. A summary of laboratory tests obtained to reach a final diagnosis.

Correct Answer: The correct answer is option b. A diagnostic path is a description of the steps taken from first presentation to the point a stable diagnosis (diagnosis upon which treatment is based) is obtained. Choice (a) better describes a diagnostic pathway-a prescriptive approach to diagnostic evaluation available in some guidelines. A diagnostic path largely reflects physicians' diagnostic practices and not patient preferences (choice c). It encompasses imaging and other diagnostic procedures, referrals, trials of medication, and follow-up intervals in addition to laboratory tests (choice d).

2. Which characteristic of a common presenting problem make it especially suitable for study through diagnostic paths?

a. The problem is extremely rare in the general population of patients.

b. Guidance on diagnostic evaluation of the problem is available in the form of evidence-based guidelines.

c. Physicians have adopted uniform approaches to diagnostic evaluation of the problem.

$\mathrm{d}$. The problem is associated with both very serious and relatively benign underlying pathology.
Correct Answer: The correct answer is option d. Identifying meaningful patterns among diagnostic paths requires significant numbers of patients, so rare problems are difficult to study (choice a). In cases in which valuable guidance is already available and when physicians have already adopted uniform practices, study of diagnostic paths is naturally less likely to prove valuable (choices b and c). The ideal type of presenting complaint is one associated with very serious underlying pathology (such as gastrointestinal cancer) and relatively benign pathology (mild gastroesophageal reflux disease). These are the types of complaints that are especially challenging and associated with substantial variation in diagnostic practices. When underlying pathology is usually benign, specific diagnostic choices are less likely to have a serious impact. When underlying pathology is usually very serious, physicians are likely to consistently adopt intense diagnostic evaluation to identify it.

\section{Protection of Human and Animal Subjects}

This study was performed in compliance with the World Medical Association Declaration of Helsinki on Ethical Principles for Medical Research Involving Human Subjects, and was reviewed and approved by the Institutional Review Board of the NorthShore University HealthSystem.

\section{Conflict of Interest}

None declared. 


\section{References}

1 National Academies of Sciences, Engineering, and Medicine. Improving Diagnosis in Health Care. Washington, DC: The National Academies Press; 2015:2

2 Saber Tehrani AS, Lee H, Mathews SC, et al. 25-Year summary of US malpractice claims for diagnostic errors 1986-2010: an analysis from the National Practitioner Data Bank. BMJ Qual Saf 2013; 22(08):672-680

3 Kim AS, Sidney S, Klingman JG, Johnston SC. Practice variation in neuroimaging to evaluate dizziness in the ED. Am J Emerg Med 2012;30(05):665-672

4 Cherkin DC, Deyo RA, Wheeler K, Ciol MA. Physician variation in diagnostic testing for low back pain. Who you see is what you get. Arthritis Rheum 1994;37(01):15-22

5 Marewski JN, Gigerenzer G. Heuristic decision making in medicine. Dialogues Clin Neurosci 2012;14(01):77-89

6 Pines JM, Isserman JA, Szyld D, Dean AJ, McCusker CM, Hollander $\mathrm{JE}$. The effect of physician risk tolerance and the presence of an observation unit on decision making for ED patients with chest pain. Am J Emerg Med 2010;28(07):771-779

7 Graber ML, Franklin N, Gordon R. Diagnostic error in internal medicine. Arch Intern Med 2005;165(13):1493-1499

8 Ely JW, Kaldjian LC, D’Alessandro DM. Diagnostic errors in primary care: lessons learned. J Am Board Fam Med 2012;25(01): 87-97

9 Song Y, Skinner J, Bynum J, Sutherland J, Wennberg JE, Fisher ES. Regional variations in diagnostic practices. N Engl J Med 2010;363 (01):45-53

10 Goldman RD, Scolnik D, Chauvin-Kimoff L, et al; Fever in Infants Group Research, Pediatric Emergency Research of Canada. Practice variations in the treatment of febrile infants among pediatric emergency physicians. Pediatrics 2009;124(02):439-445

11 Adelman A. Abdominal pain in the primary care setting. J Fam Pract 1987;25(01):27-32

12 Kerber KA, Newman-Toker DE. Misdiagnosing dizzy patients: common pitfalls in clinical practice. Neurol Clin 2015;33(03): 565-575, viii

13 Newman-Toker DE. Missed stroke in acute vertigo and dizziness: it is time for action, not debate. Ann Neurol 2016;79(01): 27-31
14 Rao G, Epner P, Bauer V, Solomonides A, Newman-Toker DE. Identifying and analyzing diagnostic paths: a new approach for studying diagnostic practices. Diagnosis (Berl) 2017;4(02):67-72

15 Zhang Y, Padman R, Epner P, Bauer V, Solomonides A, Rao G. Identifying diagnostic paths for undifferentiated abdominal pain from electronic health record data. AMIA Jt Summits Transl Sci Proc 2018;2017:290-299

16 Woodwell DA, Cherry DK. National Ambulatory Medical Care Survey: 2002 summary. Adv Data 2004;(346):1-44

17 Brekke M, Eilertsen RK. Acute abdominal pain in general practice: tentative diagnoses and handling. A descriptive study. Scand J Prim Health Care 2009;27(03):137-140

18 van den Heuvel-Janssen HA, Borghouts JA, Muris JW, Koes BW, Bouter LM, Knottnerus JA. Chronic non-specific abdominal complaints in general practice: a prospective study on management, patient health status and course of complaints. BMC Fam Pract 2006;7:12

19 Klinkman MS. Episodes of care for abdominal pain in a primary care practice. Arch Fam Med 1996;5(05):279-285

20 Lewis LM, Banet GA, Blanda M, Hustey FM, Meldon SW, Gerson LW. Etiology and clinical course of abdominal pain in senior patients: a prospective, multicenter study. J Gerontol A Biol Sci Med Sci 2005;60(08):1071-1076

21 van der Weijden T, van Bokhoven MA, Dinant GJ, van Hasselt CM, Grol RP. Understanding laboratory testing in diagnostic uncertainty: a qualitative study in general practice. Br J Gen Pract 2002; 52(485):974-980

22 Gans SL, Pols MA, Stoker J, Boermeester MA; expert steering group. Guideline for the diagnostic pathway in patients with acute abdominal pain. Dig Surg 2015;32(01):23-31

23 Caban JJ, Gotz D. Visual analytics in healthcare-opportunities and research challenges. J Am Med Inform Assoc 2015;22(02):260-262

24 Zhang Y, Padman R, Patel N. Paving the COWpath: learning and visualizing clinical pathways from electronic health record data. J Biomed Inform 2015;58:186-197

25 Perer A, Wang F, Hu J. Mining and exploring care pathways from electronic medical records with visual analytics. J Biomed Inform 2015;56:369-378

26 Gotz D, Wang F, Perer A. A methodology for interactive mining and visual analysis of clinical event patterns using electronic health record data. J Biomed Inform 2014;48:148-159 